\title{
ANÁLISIS DE UN IMPUESTO AL CO 2 SOBRE GENERADORAS TERMOELÉCTRICAS USANDO INDICADORES DE COSTO-EFECTIVIDAD
}

\author{
Analysis of $\mathrm{a} \mathrm{CO}_{2}$ tax on thermoelectric generators using cost-effectiveness indicators \\ Catalina GARCÍA NIEDMANN y Cristian MARDONES POBLETE*
}

Departamento de Ingeniería Industrial, Universidad de Concepción, Edmundo Larenas 215 4to piso, Concepción, Chile

*Autor para correspondencia: crismardones@udec.cl

(Recibido: septiembre 2018; aceptado: junio 2019)

Palabras clave: tecnología de captura, secuestro de carbono, impuesto al carbono, impuesto verde

\section{RESUMEN}

En el año 2017 se introdujo en Chile un impuesto a las emisiones de $\mathrm{CO}_{2}$ provenientes de fuentes fijas con potencia térmica $\geq 50$ Megawatts (MW). Por lo anterior, el presente estudio utiliza indicadores de costo-efectividad para evaluar si invertir en tecnología de captura y secuestro de carbono es una opción atractiva para cada una de las generadoras termoeléctricas con el objetivo de reducir sus emisiones de $\mathrm{CO}_{2}$ y su carga tributaria. Los resultados indican que el actual impuesto de 5 dólares americanos $/ \mathrm{tCO}_{2}$ es completamente inefectivo para reducir emisiones ya que las generadoras termoeléctricas sólo reducen emisiones si se fijan impuestos cercanos a 30 dólares $/ \mathrm{tCO}_{2}$. Así, se puede concluir que el impuesto actualmente aplicado en Chile sirve sólo para recaudar y no para reducir emisiones.

Key words: sequestration technology, carbon capture, carbon tax, green tax

\begin{abstract}
In 2017, Chile introduced a $\mathrm{CO}_{2}$ tax on emissions from fixed sources with a thermal power $\geq 50 \mathrm{MW}$. Therefore, this study uses cost-effectiveness indicators to assess whether investing in carbon capture and sequestration technology is an attractive option for each of the thermoelectric generators with the objective of reducing its $\mathrm{CO}_{2}$ emissions and tax burden. The results indicate that a tax of $\mathrm{US} \$ 5 / \mathrm{tCO}_{2}$ is fully infective in reducing emissions because thermoelectric generators only reduce their emissions if taxes were set at approximately US $\$ 30 / \mathrm{tCO}_{2}$. Thus, it can be concluded that the current tax only serves for collecting rather than for reducing emissions.
\end{abstract}

\section{INTRODUCCIÓN}

De acuerdo con la Organización Meteorológica Mundial las concentraciones promedio mundiales de dióxido de carbono $\left(\mathrm{CO}_{2}\right)$ alcanzaron un nuevo registro histórico con 405.5 partes por millón (ppm) en 2017 , frente a 403.3 ppm en 2016 y 400.1 ppm en 2015 (WMO 2018). Las emisiones de $\mathrm{CO}_{2}$ también 
llegaron a otro registro histórico en el año 2018 al alcanzar 37.1 gigatoneladas de acuerdo con un estudio realizado por Global Carbon Project (Le Quéré et al. 2018). Además, existe consenso científico sobre que el calentamiento global es un hecho causado por la acción del hombre y que está generando aumentos en las temperatura del planeta, deforestación, inundaciones y diversos efectos climáticos anormales. Por este motivo muchos países han formado alianzas para enfrentar este problema. En la 21 Conferencia de las Partes (COP21) de la Convención Marco de las Naciones Unidas sobre el Cambio Climático (CMNUCC), realizada en el año 2017, se alcanzó un acuerdo jurídicamente vinculante entre los países miembros de la CMNUCC para limitar el aumento de la temperatura en la superficie a no más de $2{ }^{\circ} \mathrm{C}(\mathrm{Ab}-$ dirahman y Mohd 2017). Luego de este acuerdo se le pidió al Intergovernmental Panel on Climate Change (IPCC) proveer un reporte especial el año 2018 que determinase los impactos del calentamiento global. Los resultados de este reporte muestran que las actividades humanas han incrementado la temperatura entre $0.8^{\circ} \mathrm{C}$ y $1.2^{\circ} \mathrm{C}$ sobre los niveles alcanzados en la era pre-industrial y, además, que se podría alcanzar $1.5^{\circ} \mathrm{C}$ de incremento entre 2030 y 2052 si se continúa con las tasas actuales (IPCC 2018).

Chile decidió formar parte del acuerdo comprometiéndose a reducir sus emisiones de gases de efecto invernadero (GEI) en un $30 \%$ por unidad de PIB respecto a los niveles del año 2007. Para lograr este objetivo de reducción se plantearon diferentes medidas, entre las cuales destaca la inclusión de la eficiencia energética como política de Estado y lograr que en el año 2025 el $45 \%$ de la capacidad de generación eléctrica provenga de fuentes energéticas renovables no convencionales. Además, se estableció un impuesto de 5 dólares americanos $/ \mathrm{tCO}_{2}$ para fuentes fijas con potencia térmica $\geq 50 \mathrm{MW}$ que comenzó a regir en el año 2017 (MMA 2017).

La implementación de impuestos al $\mathrm{CO}_{2}$ ha generado diversos cuestionamientos acerca de su efectividad para reducir de emisiones, ya que este impuesto podría transformarse en un instrumento de recaudación sin mejora ambiental si se fija por debajo del costo que significa para una fuente emisora ya instalada reducir emisiones más allá de las normas regulatorias ya existentes (Mardones y Flores 2017, Mardones y Flores 2018).

De esta manera el objetivo del presente estudio es evaluar los efectos ambientales de implementar impuestos al $\mathrm{CO}_{2}$ en generadoras termoeléctricas de Chile, considerando que estas podrían invertir en tecnología de captura y secuestro de carbono (CSC) para reducir emisiones. Para lo anterior, se estiman las emisiones de las generadoras termoeléctricas a partir de la información disponible en la Comisión Nacional de Energía (CNE) para el año 2017. Luego, se discuten las opciones asociadas a la inversión de tecnología para reducir emisiones en las termoeléctricas, para finalmente realizar un análisis económico con indicadores de costo-efectividad que permita encontrar la mejor alternativa que escogerían las generadoras termoeléctricas ante diferentes tasas impositivas.

\section{ANTECEDENTES}

En los últimos 40 años el consumo y la producción energética a nivel mundial se han duplicado (IEA 2017). Sin embargo, las matrices energéticas de los diferentes países han sufrido modificaciones ya que los combustibles fósiles (carbón, petróleo y gas natural) han ido perdiendo participación moderadamente para dar paso a una mayor presencia de energías renovables. Con respecto a la generación eléctrica a carbón, esta se ha mantenido como la más importante, alcanzando una participación cercana al $40 \%$. La generación con base en derivados del petróleo se ha reducido, alcanzando una participación cercana al $5 \%$ en el sector eléctrico. Al mismo tiempo ha aumentado la participación de la generación con base en energía nuclear, gas natural y energías renovables (IEA 2017).

Según datos de la CNE la capacidad instalada total en Chile en el año 2017 fue de 22540 MW, dicha capacidad se encuentra separada principalmente en el sistema interconectado central (SIC) que representa el $75.8 \%$ de la capacidad instalada total y el sistema interconectado del norte grande (SING) que representa el $23.5 \%$ de la capacidad instalada total. A su vez, los principales combustibles utilizados en el proceso de generación eléctrica fueron el carbón con un $21.2 \%$ de participación en el año 2017, el segundo lugar lo ocupó el gas natural con una participación del $20 \%$, finalmente el petróleo diésel tuvo una participación del $13.2 \%$ y la de la biomasa fue del $2 \%$. Los combustibles mencionados anteriormente fueron los encargados de producir el $55 \%$ de la electricidad en Chile durante el año 2017, mientras que las energías renovables produjeron el $45 \%$ de electricidad restante.

Uno de los subproductos negativos de la generación de electricidad es la emisión de GEI que lleva consigo a la contaminación atmosférica mundial (IPCC 2017). Para reducir las emisiones de GEI los 
países han propuesto una variedad de líneas de acción. De acuerdo con el Plan de Acción Nacional de Cambio Climático de Chile (MMA 2017) existen 30 líneas de acción en las que se está trabajando actualmente (ver Anexo B). Algunas medidas específicas tienen como objetivo la promoción de generación de energía a través de fuentes renovables que permiten no sólo la reducción de las emisiones, sino que también permiten disminuir la dependencia de combustibles fósiles. Esto es particularmente relevante para los países que son importadores de combustibles fósiles, como es el caso de Chile. Otras medidas adoptadas por distintos países hacen referencia a la incorporación de impuestos al $\mathrm{CO}_{2}$ con el fin de incentivar la incorporación de nuevas tecnologías de mayor eficiencia y generar menos emisiones atmosféricas (González-Díaz et al. 2017).

Actualmente, existen distintas alternativas para reducir las emisiones de $\mathrm{CO}_{2}$ en las generadoras termoeléctricas, algunas de ellas son exclusivas para plantas nuevas ya que no es posible adaptar las tecnologías para las generadoras existentes, como la gasificación del carbón y la gasificación subterránea del carbón. Existen en cambio otras alternativas como la captura y secuestro del carbono (CSC) que es posible utilizar en generadoras ya existentes con algunas modificaciones en calderas y quemadores. Sin embargo, no es posible aumentar la eficiencia de las generadoras térmicas. El sistema de CSC se divide principalmente en tres fases: captura, transporte y almacenamiento (Abdirahman et al. 2018). A su vez, existen tres opciones para capturar el $\mathrm{CO}_{2}$, estas son la captura en pre combustión, oxicombustión y postcombustión, la selección de estas tecnologías depende de las características propias de cada central termoeléctrica (GCCSI 2017).

La captura de $\mathrm{CO}_{2}$ en precombustión consiste en producir una mezcla gaseosa compuesta principalmente de hidrógeno y $\mathrm{CO}_{2}$ (proveniente de la gasificación del carbón y otros hidrocarburos) para posteriormente separar estos dos gases. La separación se basa en la descarbonización del combustible antes de la combustión mediante técnicas de gasificación del carbón o reformando el gas natural (Coraliae 2015, Valiani et al. 2017). Una vez separados los gases es posible capturar el $\mathrm{CO}_{2}$ y prepararlo para el transporte y almacenamiento, mientras que el gas combustible rico en hidrógeno se puede utilizar para las turbinas a gas (CCCEP 2015). La captura de $\mathrm{CO}_{2}$ en postcombustión se basa en un sistema de separación de $\mathrm{CO}_{2}$ desde los gases producidos por la combustión de un combustible primario con aire. En lugar de ser liberados a la atmósfera, los gases de escape son sometidos a un proceso de remoción del $\mathrm{CO}_{2}$, producto que es enviado a un lugar de almacenamiento. Entre los procesos más viables para capturar el $\mathrm{CO}_{2}$ se encuentran el ciclo de calcinación/ carbonatación y la absorción química con aminas (Valencia y Cardona 2013, Petrescu et al. 2017). La captura del $\mathrm{CO}_{2}$ en oxicombustión se realiza durante la combustión y tiene un largo recorrido como tecnología aplicada. Básicamente consiste en la utilización de oxígeno en lugar de aire para la combustión. Esta tecnología es utilizada en centrales de nueva generación con ciclos agua-vapor extremadamente críticos, así como también, en turbinas de gas con o sin calderas de recuperación (Consoli y Wildgust 2017, López et al. 2018).

Algunos estudios han intentado determinar los costos asociados a la incorporación de tecnologías de CSC en termoeléctricas. Hu y Zhai (2017) estimaron el costo nivelado de la electricidad (LCOE) y el costo de abatimiento de $\mathrm{CO}_{2}$ en China para termoeléctricas a carbón con capacidad de generación neta de 1.238 MW. De acuerdo con sus estimaciones, la implementación de CSC en plantas termoeléctricas a carbón disminuiría la eficiencia neta de la planta en $11 \%$, y además, aumentarían los costos totales de capital y el LCOE de la planta en un $81 \%$ y un $73 \%$, respectivamente. A su vez, el costo de $\mathrm{CO}_{2}$ evitado sería de aproximadamente 41 dólares $/ \mathrm{tCO}_{2}$. Otro estudio realizado por GCCSI (2017) estimó los costos asociados a las tecnologías de CSC en las fuentes generadoras de electricidad que se encuentran en funcionamiento, los cuales varían dependiendo de las distintas tecnologías y combustibles utilizados. En el cuadro I se muestra un resumen de ciertos parámetros y costos asociados a la incorporación de tecnologías de CSC para termoeléctricas a carbón y a gas natural de acuerdo con el estudio previamente mencionado. En una termoeléctrica a carbón el costo de producir electricidad sin tecnología de captura se encuentra en el orden de 75-77 dólares/MWh, mientras que al incorporar la tecnología de CSC los costos se incrementan a 124-133 dólares/MWh. Además, el costo de mitigación del $\mathrm{CO}_{2}$ es aproximadamente 74-83 dólares $/ \mathrm{tCO}_{2}$, mientras que para el caso de una termoeléctrica a gas natural el costo de abatir es aproximadamente 89 dólares $/ \mathrm{tCO}_{2}$. Adicionalmente, es necesario agregar el costo de transporte y almacenamiento que se encuentran en el orden de 7-12 dólares $/ \mathrm{tCO}_{2}$ (GCCSI 2017).

Por otra parte, la incorporación de impuestos a las emisiones de $\mathrm{CO}_{2}$ en Chile traería consigo un aumento en los precios de la electricidad, se estima que el incremento podría variar entre $0.4 \%$ a $0.7 \%$ por cada dólar de impuesto (Benavides et al. 2015, 
CUADRO I. COMPARACIÓN DE COSTOS DE CAPTURA Y SECUESTRO DE CARBONO (CSC) PARA GENERADORAS TERMOELÉCTRICAS A CARBÓN Y GAS NATURAL

\begin{tabular}{|c|c|c|c|c|}
\hline & $\begin{array}{l}\text { Carbón } \\
\text { sin CSC }\end{array}$ & $\begin{array}{l}\text { Carbón } \\
\text { con CSC }\end{array}$ & $\begin{array}{c}\text { Gas natural } \\
\text { sin CSC }\end{array}$ & $\begin{array}{c}\text { Gas natural } \\
\text { con CSC }\end{array}$ \\
\hline Potencia bruta (MW) & 580 & 642 & 641 & 601 \\
\hline Eficiencia planta (\%) & 40.7 & 32.5 & 51.5 & 45.7 \\
\hline Generación de $\mathrm{CO}_{2}(\mathrm{t} / \mathrm{h})$ & 426 & 534 & 225 & 225 \\
\hline $\mathrm{CO}_{2}$ emitido $(\mathrm{t} / \mathrm{h})$ & 426 & 53 & 225 & 22 \\
\hline $\mathrm{CO}_{2}$ capturado $(\mathrm{t} / \mathrm{h})$ & 0 & 480 & 0 & 202 \\
\hline Intensidad de emisión (kg/MWh) & 774 & 97 & 356 & 40 \\
\hline Costo capital equipamiento (Mill dólares) & 556 & 865 & 243 & 365 \\
\hline Costo capital materiales (Mill dólares) & 56 & 136 & 28 & 77 \\
\hline Costo capital puesta en marcha (Mill dólares) & 352 & 588 & 83 & 201 \\
\hline Costos extras (Mill dólares) & 223 & 475 & 79 & 214 \\
\hline Costos totales (Mill dólares) & 1187 & 2065 & 432 & 856 \\
\hline Costos totales (dólares/kW) & 2158 & 3754 & 686 & 1531 \\
\hline Costos variables de operación y mantención (dólares/MWh) & 9.64 & 17.84 & 1.18 & 2.3 \\
\hline
\end{tabular}

Fuente: GCCSI (2017). CSC = captura y secuestro de carbono, MW = megawatt, $\mathrm{t} / \mathrm{h}=$ tonelada por hora, $\mathrm{kg} / \mathrm{MWh}=\mathrm{kilogramo}$ por megawatt por hora, Mill $=$ millones, $\mathrm{kW}=$ kilowatt

Vera y Sauma 2015, Mardones y Muñoz 2017, Mardones y Baeza 2018).

\section{DESARROLLO}

\section{Tratamiento de información y estimación de emisiones}

La CNE genera informes mensuales que incluyen datos estadísticos del sector eléctrico tales como capacidades de generación eléctrica, precios de la electricidad y niveles de producción. Para obtener datos sobre el consumo de combustibles y emisiones de las generadoras termoeléctricas se analizaron las bases de datos "Capacidad instalada de generación" y "Consumo combustibles" de la CNE del año 2017. De esta manera, se obtuvo información sobre el tipo de sistema, región, tipo de unidad, combustible utilizado, consumo de combustible, potencia térmica, entre otros. Es necesario mencionar que se excluyeron las generadoras hidroeléctricas, solares y eólicas ya que el análisis se realizó con base en las termoeléctricas sujetas a impuestos. Así, se contabilizaron 176 unidades generadoras termoeléctricas a lo largo del país. Sin embargo, el $26 \%$ de esas unidades generadoras tenían una capacidad instalada menor a 2 MW por lo cual no se les aplica el impuesto al $\mathrm{CO}_{2}$, o bien, no se especificaba el consumo de combustible, por lo que finalmente se incluyeron en el análisis sólo 129 unidades generadoras termoeléctricas.

Con respecto al consumo de combustible de cada unidad generadora, en la base de datos "Consumo de combustible" se reportaba el consumo mensual y anual de cada termoeléctrica. En muchos casos fue necesaria la trasformación de unidades para poder comparar los consumos según los tipos de combustibles. En general para el gas natural, petróleo diésel y petróleo combustible se utilizó la unidad de medida $\mathrm{m}^{3}$, mientras que para el carbón se utilizó la unidad de medida tonelada.

Para la estimación de la energía consumida fue necesaria la utilización de la cantidad de combustible, la densidad del combustible y su poder calorífico. Las densidades de cada combustible junto a su poder calorífico se reportan en el cuadro II.

Luego, se estimó el consumo energético de cada combustible en millones de BTU (MMBTU) con el fin de que cada fuente generadora presentara la misma unidad y así facilitar la estimación de emisiones. Para esto, se utilizó el factor de conversión que indica que 1 BTU corresponde a 252 calorías.

En las ecuaciones (1) y (2) se indica el procedimiento para obtener el consumo energético.

$$
\begin{aligned}
& \text { Energía }(\text { kcal })=\text { cantidad combustible } \\
& \qquad(\text { unidad }) * \text { poder calorifico }\left(\frac{k c a l}{\text { unidad }}\right)
\end{aligned}
$$

$$
\begin{aligned}
& \text { Energía }(B T U)=\text { Energía }(\text { kcal }) * \\
& \frac{1000 \mathrm{Cal}}{1 \mathrm{kcal}} * \frac{1 \mathrm{BTU}}{252 \mathrm{Cal}}
\end{aligned}
$$

Con los factores de emisión de $\mathrm{CO}_{2}$ que se encuentran en el cuadro III y los consumos de energía 
CUADRO II. PODER CALORÍFICO Y DENSIDAD DE COMBUSTIBLES

\begin{tabular}{lcc}
\hline Combustible & $\begin{array}{c}\text { Poder calorífico } \\
\text { inferior }\end{array}$ & Densidad \\
\hline Carbón & $7000 \mathrm{kcal} / \mathrm{kg}$ & $750 \mathrm{~kg} / \mathrm{m}^{3}$ \\
Coque de petróleo & $7000 \mathrm{kcal} / \mathrm{kg}$ & $750 \mathrm{~kg} / \mathrm{m}^{3}$ \\
Petróleo diésel & $10.9 \times 10^{6} \mathrm{kcal} / \mathrm{m}^{3}$ & $840 \mathrm{~kg} / \mathrm{m}^{3}$ \\
Petróleo combustible & $10.5 \times 10^{6} \mathrm{kcal} / \mathrm{m}^{3}$ & $944 \mathrm{~kg} / \mathrm{m}^{3}$ \\
Biomasa & $3500 \mathrm{kcal} / \mathrm{kg}$ & $500 \mathrm{~kg} / \mathrm{m}^{3}$ \\
Gas natural & $9341 \mathrm{kcal} / \mathrm{kg}$ & $673 \mathrm{~kg} / \mathrm{m}^{3}$ \\
Gas licuado & $12100 \mathrm{kcal} / \mathrm{kg}$ & $550 \mathrm{~kg} / \mathrm{m}^{3}$ \\
\hline
\end{tabular}

Fuente: Ministerio de Energía (2017)

CUADRO III. FACTORES DE EMISIÓN DE $\mathrm{CO}_{2}$ PARA DISTINTOS TIPOS DE COMBUSTIBLES

\begin{tabular}{lcc}
\hline Combustible & Factor & $\begin{array}{c}\text { Unidad (Millón de } \\
\left.\mathrm{BTU}=10^{6} \mathrm{BTU}\right)\end{array}$ \\
\hline Petróleo diésel & 77.2 & $\mathrm{~kg} \mathrm{de} \mathrm{CO}_{2} /$ Millón de BTU \\
Petróleo combustible & 77.2 & $\mathrm{~kg} \mathrm{de} \mathrm{CO}_{2} /$ Millón de BTU \\
Carbón & 97.6 & $\mathrm{~kg} \mathrm{de} \mathrm{CO}_{2} /$ Millón de BTU \\
Gas natural & 58.8 & $\mathrm{~kg} \mathrm{de} \mathrm{CO}_{2} /$ Millón de BTU \\
Biomasa & 0 & $\mathrm{~kg} \mathrm{de} \mathrm{CO}_{2} /$ Millón de BTU \\
Gas licuado & 65.7 & ${\mathrm{~kg} \mathrm{de} \mathrm{CO}_{2} / \text { Millón de BTU }}$ \\
\hline
\end{tabular}

Fuente: SMA (2016). BTU = unidad térmica británica

por uso de combustible obtenidos anteriormente, se estimaron las emisiones anuales de cada fuente generadora termoeléctrica según la siguiente ecuación.

Emisiones $\left(\mathrm{Kg} \mathrm{CO}_{2}\right)=$ Factor emisión

$$
\left(\frac{\mathrm{KgCO}}{\mathrm{MMBTU}}\right) * \text { energía }(\mathrm{MMBTU})
$$

En el cuadro IV se observa la clasificación de las termoeléctricas según los tipos de combustibles utilizados, consumo de combustible y generación anual de energía.
Al analizar las emisiones de las 129 termoeléctricas pertenecientes al SIC y SING se estimó que el total de emisiones asociadas a la producción de energía en el año 2017 fue de 32 millones de $\mathrm{tCO}_{2}$. Específicamente, el $98 \%$ de emisiones corresponden al consumo de carbón para la generación de energía y el $2 \%$ restante se asocia al consumo de petróleo diésel, petróleo combustible y gas natural. En el cuadro $\mathbf{V}$ se muestran las emisiones según tipo de combustible.

CUADRO V. EMISIONES SEGÚN TIPOS DE COMBUSTIBLES EN TERMOELÉCTRICAS

\begin{tabular}{lc}
\hline Combustible & Emisiones $\left(\mathrm{tCO}_{2}\right)$ \\
\hline Carbón/petcoke & 32110572 \\
Gas natural & 14038 \\
Petróleo diésel & 325239 \\
Petróleo combustible & 31084
\end{tabular}

Fuente: Elaboración propia a partir de datos obtenidos por CNE (2017)

\section{Costos de tecnología CSC}

Una vez estimadas las emisiones de cada unidad generadora termoeléctrica fue necesario analizar los distintos escenarios a los que se enfrentarían con base en los impuestos al $\mathrm{CO}_{2}$ aplicados. Para poder hacer efectivo el análisis y de acuerdo con los distintos estudios sobre las tecnologías de reducción de emisiones, se seleccionó la tecnología de CSC en postcombustión ya que según la bibliografía analizada es posible incorporar esta tecnología a una unidad generadora en funcionamiento.

Para poder analizar el comportamiento de las generadoras termoeléctricas frente a los distintos escenarios impositivos fue necesario estimar los costos asociados al consumo de combustibles sin cambios

CUADRO IV. GENERADORAS TÉRMICAS ASOCIADAS A COMBUSTIBLES FÓSILES

\begin{tabular}{lccc}
\hline Combustible & $\begin{array}{c}\text { Cantidad } \\
\text { termoeléctricas }\end{array}$ & $\begin{array}{c}\text { Consumo } \\
\text { combustible }\end{array}$ & $\begin{array}{c}\text { Generación } \\
\text { anual (MWh) }\end{array}$ \\
\hline Carbón/petcoke & 29 & 11844063 ton & 29424313 \\
Gas natural & 20 & $6436733 \mathrm{~m}^{3}$ & 11855700 \\
Petróleo diésel & 73 & $97400 \mathrm{~m}^{3}$ & 287354 \\
Petróleo combustible & 7 & $9908 \mathrm{~m}^{3}$ & 43760 \\
\hline
\end{tabular}

Fuente: Elaboración propia a partir de datos obtenidos por CNE (2017) 
tecnológicos. Para esto se utilizaron los datos de los precios de combustibles indicados en el cuadro VI.

\section{CUADRO VI. PRECIO DE COMBUSTIBLES}

\begin{tabular}{lc}
\hline Combustible & Precio dólares/Millón de BTU \\
\hline Carbón & 4.25 \\
Petróleo diésel & 10.91 \\
Petróleo combustible & 7.78 \\
Gas natural & 3.0 \\
\hline
\end{tabular}

Fuente: Elaboración propia a partir de datos obtenidos por CNE (2017).

Para obtener los costos asociados a la opción tecnológica de $\mathrm{CSC}$ de $\mathrm{CO}_{2}$ para generadoras termoeléctricas existentes se utilizaron datos de distintos estudios reportados en el cuadro A.I, cuadro A.II y cuadro A.III del Anexo, que a su vez sirvieron para estimar los costos para la tecnología de CSC a partir de una función de costos descrita por Rubin et al. (2012), la cual evalúa los costos asociados a la tecnología de CSC a través del costo nivelado de la electricidad (LCOE) y el costo de abatimiento de $\mathrm{CO}_{2}$. A partir de la información sobre costos asociados a la CSC reportados en el Anexo se generó una nueva función de costos anualizados que depende de la potencia térmica en MW de la generadora termoeléctrica utilizando 31 observaciones que arrojó un coeficiente de determinación o $\mathrm{R}^{2}$ igual a 0.99 .

$$
\begin{aligned}
& \text { Costo abatimiento }\left(\frac{U S \$}{t C O 2}\right)= \\
& \exp 0.6028966 * \ln (\text { Potencia } M W)+0.7075519
\end{aligned}
$$

Es importante mencionar que la ecuación (4) representa el costo anualizado de abatimiento por 1 $\mathrm{tCO}_{2}$, por lo que para obtener el costo total de abatimiento anual es necesario multiplicar la ecuación por el total de toneladas abatidas en un año en el caso de utilizar la tecnología de CSC. También es necesario mencionar que los distintos estudios utilizados para estimar la función de costo de abatimiento consideran generadoras termoeléctricas mayores a $100 \mathrm{MW}$ de potencia térmica instalada, por lo cual no son válidos para potencias menores.

Además, la eficiencia de una planta con tecnología de CSC disminuye en promedio un $10 \%$ en comparación a una generadora termoeléctrica sin la opción tecnológica (Rochedo et al. 2016, Carapellucci et al. 2017). De esta manera, la reducción de eficiencia se traduce en un aumento del consumo de combustible para lograr la misma potencia de salida para los dos escenarios. Una vez estimados los nuevos consumos de combustibles, se obtuvieron los costos asociados al consumo de combustible y pago de impuestos de sus emisiones, asumiendo una eficiencia de captura de $\mathrm{CO}_{2}$ del $90 \%$ (Carapellucci et al. 2017, Van der Spek et al. 2017).

Finalmente, fue necesario considerar el aumento en los ingresos de las generadoras termoeléctricas asociados al incremento en los precios de la electricidad luego de la aplicación de impuestos al $\mathrm{CO}_{2}$. Benavides et al. (2015) estimaron que por cada dólar de impuesto asociado a las $\mathrm{tCO}_{2}$ emitidas aumentarían los precios de la electricidad en un $0.4 \%$. Mardones y Muñoz (2017) estimaron que el incremento en los precios sería de $0.6 \%$ por cada dólar de impuesto, mientras que Vera y Sauma (2015) concluyeron que el aumento en los precios sería de $0.7 \%$. En este estudio se estimaron los ingresos extras asociados al aumento en los precios de la electricidad basándose en el promedio de los tres estudios mencionados anteriormente, es decir, asumiendo un aumento del $0.6 \%$ en los precios de la electricidad por cada dólar de impuesto a las emisiones de $\mathrm{CO}_{2}$.

\section{Comparación de alternativas con base en indica- dores de costo-efectividad}

En este estudio se comparan las alternativas de pagar impuestos o capturar emisiones a partir de indicadores de costo-efectividad. Esta metodología consiste en comparar los costos de ambas alternativas por tonelada de $\mathrm{CO}_{2}$ y escoger aquella que resultaría menos costosa para cada generadora termoeléctrica. Así, con los datos de los costos netos anualizados asociados a las tecnologías de CSC fue posible simular el comportamiento de las generadoras termoeléctricas ante la implementación de impuestos a las emisiones de $\mathrm{CO}_{2}$. Cabe destacar que se consideró la posibilidad de que cada fuente termoeléctrica podía mantener su situación base de no incorporar la tecnología de CSC y pagar impuestos o bien incorporar la tecnología de captura de $\mathrm{CO}_{2}$ y reducir el pago de impuestos asociado a las emisiones.

Así, se realizó una matriz de comparación con indicadores de costo-efectividad donde estaba presente el escenario actual en el cual no existía la opción de invertir en tecnología de CSC, pero sí el costo del impuesto por toneladas anuales de $\mathrm{CO}_{2}$ emitidas según el tipo de generadora termoeléctrica. A modo de ejemplo se presenta en el cuadro A.IV del Anexo los cálculos de costo-efectividad para cada una de las dos alternativas que enfrenta una muestra seleccionada 
de generadoras termoeléctricas. Estas opciones son no instalar tecnología CSC pagando impuestos por sus emisiones totales, o bien, instalar tecnología CSC pagando impuestos sólo por sus emisiones no abatidas.

Para evaluar el comportamiento de las generadoras termoeléctricas ante la aplicación de impuestos a las emisiones de $\mathrm{CO}_{2}$ se consideraron distintos montos del impuesto $(1,5,10,15,20,25$ y 30 dólares americanos). Se escogieron estas tasas impositivas para evaluar el comportamiento de variar gradualmente los impuestos, y además, 5 dólares $/ \mathrm{tCO}_{2}$ corresponde al impuesto que se aplica actualmente en Chile para fuentes fijas con potencia térmica $\geq 50 \mathrm{MW}$. Mientras que el impuesto de 30 dólares/ $/ \mathrm{tCO}_{2}$ corresponde al costo social ${ }^{1}$ del $\mathrm{CO}_{2}$ utilizado en Chile por el Ministerio de Desarrollo Social.

\section{RESULTADOS Y DISCUSIÓN}

Se evaluaron 129 unidades de generación termoeléctrica, las cuales consumen distintos tipos de combustibles y emiten aproximadamente 32 millones de toneladas de $\mathrm{CO}_{2}$ al año. Como se mencionó anteriormente, para estimar el costo de incorporar la tecnología de CSC fue necesario estimar una función de costo de abatimiento de $\mathrm{CO}_{2}$, la cual se obtuvo de acuerdo con distintos estudios bibliográficos. A su vez, fue necesario estimar el nuevo consumo de combustible asociado a la disminución de eficiencia de las plantas al incorporar la tecnología de CSC. Además, se simularon dos escenarios para las generadoras termoeléctricas, el primero no considera el incremento en los precios de la electricidad al incorporar impuestos a las emisiones de $\mathrm{CO}_{2}$ y el segundo escenario considera el incremento en los precios de la electricidad $(0.6 \%$ en los precios de la electricidad por cada dólar de impuesto).

En el cuadro VII se observan los resultados de las generadoras termoeléctricas al simular el escenario en el cual no se incrementan los precios de la electricidad luego de la aplicación de diferentes tasas impositivas. Cabe destacar que para tasas impositivas entre 1 dólar/ $/ \mathrm{tO}_{2}$ y 25 dólares $/ \mathrm{tCO}_{2}$ ninguna termoeléctrica decide incorporar la tecnología de CSC y el impuesto sólo funciona como un sistema de recaudación. Esto sucede porque el costo anualizado de incorporar la tecnología de CSC para cada una de las generadoras termoeléctricas es mayor que el costo de pagar el impuesto. De esta manera, las emisiones de $\mathrm{CO}_{2}$ se mantienen en 32 millones de toneladas al aplicar impuestos de hasta 25 dólares $/ \mathrm{tCO}_{2}$.

$\mathrm{Al}$ incorporar impuestos de 30 dólares $/ \mathrm{tCO}_{2}$ se observa que 17 generadoras termoeléctricas deciden

CUADRO VII. SIMULACIÓN DE DISTINTOS IMPUESTOS A LAS EMISIONES DE $\mathrm{CO}_{2}$ EN GENERADORAS TERMOELÉCTRICAS SIN INCREMENTO EN LOS PRECIOS DE LA ELECTRICIDAD

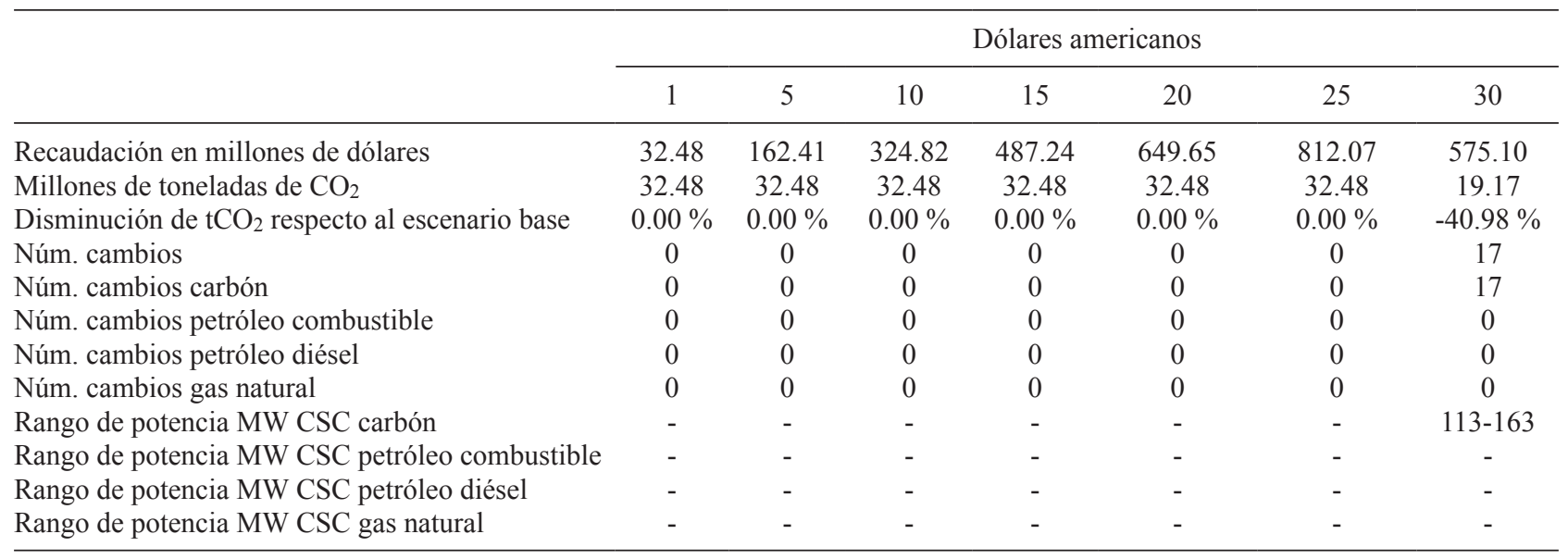

Fuente: Elaboración propia. $\mathrm{CSC}=$ captura y secuestro de carbono

\footnotetext{
${ }^{1} \mathrm{El}$ costo social del $\mathrm{CO}_{2}$ mide la magnitud de la externalidad que debe ser incorporada en los costos de producción de las empresas o bien en las decisiones sobre política e inversión pública. En teoría este costo señala cuánto estaría dispuesto a pagar la sociedad hoy para evitar un daño futuro causado por una tonelada de $\mathrm{CO}_{2}$ adicional.
} 
incorporar la tecnología de CSC, lo que significa una reducción del $40.98 \%$ de emisiones de $\mathrm{CO}_{2}$ respecto al escenario base. Bajo este escenario el $13.17 \%$ de las generadoras termoeléctricas deciden incorporar la tecnología y todas ellas utilizan carbón como combustible. El comportamiento que tienen las unidades generadoras a carbón se puede explicar por su gran aporte a las emisiones de $\mathrm{CO}_{2}$, ya que sólo las 17 generadoras termoeléctricas que deciden incorporar la tecnología emiten en condiciones normales 18 millones de $\mathrm{tCO}_{2}$ al año equivalentes al $55.4 \%$ de las emisiones totales del escenario base. Es importante mencionar que las generadoras termoeléctricas que deciden incorporar la tecnología de CSC poseen una capacidad instalada entre 113-163 MW.

En el cuadro VIII se observan los principales resultados de las generadoras termoeléctricas al simular el segundo escenario en el cual se asume un incremento en los precios de la electricidad ante la aplicación de diferentes tasas impositivas a las emisiones de $\mathrm{CO}_{2}$. Cabe destacar que para tasas impositivas entre 1 dólar/ $\mathrm{tCO}_{2}$ y 5 dólares $/ \mathrm{tCO}_{2}$ ninguna generadora termoeléctrica decide incorporar la tecnología de CSC y las emisiones se mantienen en 32.48 millones de $\mathrm{tCO}_{2}$. La aplicación de un impuesto de 15 dólares $/ \mathrm{tCO}_{2}$ induce que cinco unidades generadoras termoeléctricas a gas natural decidan incorporar la tecnología de CSC, pero la incorporación de tecnología de CSC para estas generadoras termoeléctricas sólo genera reducciones marginales en las emisiones totales. Lo anterior se explica porque las emisiones asociadas a estas generadoras termoeléctricas a gas natural son muy bajas, aportan menos del $1 \%$ de las emisiones de $\mathrm{CO}_{2}$ totales.

$\mathrm{Al}$ aplicar un impuesto de 20 dólares $/ \mathrm{tCO}_{2}$ se observa que 21 generadoras termoeléctricas deciden incorporar la tecnología de CSC. Esto se explica porque bajo este escenario aumentan los precios de la electricidad de acuerdo con los impuestos establecidos. Este aumento en los precios se traduce en un ingreso extra para las generadoras termoeléctricas, situación que no se da en el primer escenario (Cuadro VII). La incorporación de tecnología de CSC para estas fuentes genera una reducción de emisiones del $33.19 \%$ equivalente a 10.78 millones de $\mathrm{tCO}_{2}$ anuales respecto al escenario base. Es necesario mencionar que la reducción de emisiones se debe a la instalación de tecnología de CSC en las generadoras termoeléctricas que utilizan carbón. Al aplicar un impuesto de 30 dólares $/ \mathrm{tCO}_{2}$ se observa que 25 generadoras termoeléctricas a carbón deciden incorporar la tecnología, lo que genera una reducción de emisiones de 25.38 millones de $\mathrm{tCO}_{2}$ anuales respecto al escenario base.

Si se comparan los resultados de ambos escenarios (Cuadro VII y Cuadro VIII) se concluye que bajo el escenario en el cual se asume un incremento en los precios de la electricidad se genera una reducción mayor de emisiones que en el escenario en el cual no se incrementan los precios. Sin embargo, esta reducción se produce al aplicar impuestos desde los 20 dólares/ $/ \mathrm{tCO}_{2}$.

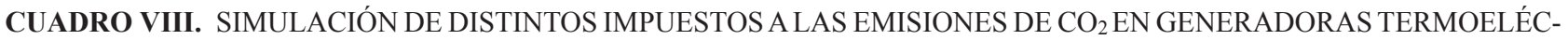
TRICAS CON INCREMENTO EN LOS PRECIOS DE LA ELECTRICIDAD

\begin{tabular}{|c|c|c|c|c|c|c|c|}
\hline & \multicolumn{7}{|c|}{ Dólares americanos } \\
\hline & 1 & 5 & 10 & 15 & 20 & 25 & 30 \\
\hline Recaudación en millones de dólares & 32.48 & 162.40 & 324.82 & 487.23 & 434.10 & 267.54 & 213.00 \\
\hline Millones de toneladas de $\mathrm{CO}_{2}$ & 32.48 & 32.48 & 32.48 & 32.48 & 21.70 & 10.70 & 7.10 \\
\hline Disminución de $\mathrm{tCO}_{2}$ respecto al escenario base & $0.00 \%$ & $0.00 \%$ & $0.00 \%$ & $0.00 \%$ & $-33.19 \%$ & $-67.06 \%$ & $-78.14 \%$ \\
\hline Núm. cambios & 0 & 0 & 4 & 5 & 21 & 29 & 31 \\
\hline Núm. cambios carbón & 0 & 0 & 0 & 0 & 15 & 23 & 25 \\
\hline Núm. cambios petróleo combustible & 0 & 0 & 0 & 0 & 0 & 0 & 0 \\
\hline Núm. cambios petróleo diésel & 0 & 0 & 0 & 0 & 1 & 1 & 1 \\
\hline Núm. cambios gas natural & 0 & 0 & 4 & 5 & 5 & 5 & 5 \\
\hline Rango de potencia MW CSC carbón & - & - & - & - & $113-161$ & $113-252$ & $113-252$ \\
\hline Rango de potencia MW CSC petróleo combustible & - & - & - & - & - & - & - \\
\hline Rango de potencia MW CSC petróleo diésel & - & - & - & - & 378 & 378 & 378 \\
\hline Rango de potencia MW CSC gas natural & - & - & $121-127$ & $121-354$ & $121-354$ & $121-354$ & $121-354$ \\
\hline
\end{tabular}

Fuente: Elaboración propia. $\mathrm{CSC}=$ captura y secuestro de carbono 


\section{CONCLUSIONES}

A partir de los resultados de este estudio se puede concluir que el impuesto de 5 dólares $/ \mathrm{tCO}_{2}$ que se aplica actualmente en Chile sirve como sistema de recaudación más que como medida efectiva para la reducción de emisiones ya que los principales beneficios ambientales se observan a partir de la aplicación de impuestos de 20 dólares $/ \mathrm{tCO}_{2}$ considerando el escenario que asume un incremento en los precios de la electricidad, o alternativamente, a partir de la aplicación de impuestos de 30 dólares $/ \mathrm{tCO}_{2}$ considerando el escenario en que se mantienen constantes los precios de la electricidad. Lo anterior, se explica porque aplicar impuestos al $\mathrm{CO}_{2}$ por debajo del costo que significa para una fuente invertir en tecnologías de abatimiento lleva a que esta política ambiental sea inefectiva para reducir emisiones. En términos simples esta inefectividad se genera porque desde un punto de vista económico una generadora termoeléctrica obligada a pagar impuestos nunca escogerá reducir sus emisiones mientras la tasa de impuesto por tonelada sea más baja que el costo de reducir esa tonelada con alguna tecnología de captura. Además, el incremento en los precios de la electricidad generados por el incremento en los costos marginales de producción permite traspasar parte del costo de la externalidad a los consumidores, lo cual ayuda a que las firmas tengan menores costos netos anualizados si instalan una tecnología de CSC incentivando su uso.

También se puede mencionar que si el impuesto ambiental se fija igual al costo social del $\mathrm{CO}_{2}$ utilizado en Chile por el Ministerio de Desarrollo Social (30 dólares/ $\mathrm{tCO}_{2}$ ) es posible observar en el escenario en el que se asume un incremento en los precios de la electricidad que 31 unidades generadoras incorporan tecnologías de CSC, lo que trae consigo una reducción de un $78 \%$ en las emisiones totales de $\mathrm{CO}_{2}$ respecto al escenario base, mientras que en el escenario sin incremento en los precios de la electricidad se observa que solo 17 unidades generadoras a carbón deciden incorporar la tecnología de CSC, lo que genera una reducción de $41 \%$ en las emisiones totales. No obstante, en ambos escenarios se observan reducciones relevantes en las emisiones de $\mathrm{CO}_{2}$ por lo cual se sugiere elevar sustancialmente el monto actual del impuesto aplicado en Chile de tal forma que se aproxime idealmente a su costo social.

\section{AGRADECIMIENTOS}

El autor para correspondencia agradece al Fondo Nacional de Desarrollo Científico y Tecnológico
(Fondecyt Regular 1191303) por el financiamiento otorgado a esta investigación.

\section{REFERENCIAS}

Abdirahman M.A., Mohd W.M., Saleh Y.A. y Mamunu M. (2018). Harnessing flexibility potential of flexible carbon capture power plants for future low carbon power systems: Review. Renew. Sust. Energ. Rev. 81 (2), 3101-3110. DOI: 10.1016/j.rser.2017.08.085

Abdirahman M.A. y Mohd W.M. (2017). Carbon capture power plants: Decoupled emission and generation outputs for economic dispatch. Int. J. Greenh. Gas. Con. 63 (1), 12-19. DOI: 10.1016/j.ijggc.2017.05.001

Akash A.R., Rao A.B. y Chandel M.K. (2016). Prospects of implementing $\mathrm{CO}_{2}$ capture and sequestration (CCS) in the proposed supercritical coal power plants in India. Enrgy. Proced. 90 (1), 604-612.

DOI: 10.1016/j.egypro.2016.11.229

Al-Qayim K., Nimo W. y Pourkashanian M. (2015). Comparative techno-economic assessment of biomass and coal with CCS technologies in a pulverized combustion power plant in the United Kingdom. Int. J. Greenh. Gas. Con. 43 (1), 82-92.

DOI: $10.1016 /$ j.ijggc.2015.10.013

AP (2011). Cost assessment of fossil power plant equipped with CCS under typical scenarios. Reporte. Alstom Power. Saint-Ouen, Francia, 22 pp.

Benavides C., Gonzales L., Diaz M., Fuentes R., García G., Palma-Behnke R. y Ravizza C. (2015). The impact of a carbon tax on the Chilean electricity generation sector. Energies 8 (4), 2674-2700. DOI: 10.3390/en8042674

Carapellucci R., Giordano L. y Vaccarelli M. (2017). Application of an amine-based $\mathrm{CO}_{2}$ capture system in retrofitting combined gas-steam power plants. Energy 118 (1), 808-826.

DOI: $10.1016 /$ j.energy.2016.10.114

CCCEP (2015). Bridging the gap: improving the economic and policy framework for carbon capture and storage in the European Union. Reporte. Center for Climate Change Economics and Policy. Londres, Reino Unido, $20 \mathrm{pp}$.

CNE (2017). Reporte capacidad instalada generación. Reporte. Comisión Nacional de Energía. Santiago, Chile, 1 pp.

Consoli C.P. y Wildgust N. (2017). Current status of global storage resources. Enrgy. Proced. 114 (1), 4623-4628.

DOI: 10.1016/j.egypro.2017.03.1866

Coraliae (2015). Potencial de captura y almacenamiento de $\mathrm{CO}_{2}$. Reporte. Coralia Environmental. Buenos Aires, Argentina, 62 pp. 
EPRI (2013). Program on technology innovation: Integrated generation technology options 2012. Reporte. Electric Power Research Institute. California, EUA, $110 \mathrm{pp}$.

GCCSI (2011). Economic assessment of carbon capture and storage technologies. Reporte. Global CCS Institute. Canberra, Australia, $60 \mathrm{pp}$.

GCCSI (2017). The global status of CCS: 2017. Reporte. Global CCS Institute. Australia, 43 pp.

González-Díaz A., González-Díaz M.O., AlcarázCalderóna A.M., Gibbins J. y Lucquiaud M. (2017). Priority projects for the implementation of CCS power generation with enhanced oil recovery in Mexico. Int. J. Greenh. Gas. Con. 64 (1), 119-125.

DOI: 10.1016/j.ijggc.2017.07.006

Hanak D.P. y Manovic V. (2018). Techno-economic feasibility assessment of $\mathrm{CO}_{2}$ capture from coal-fired power plants using molecularly imprinted polymer. Fuel 214 (1), 512-520. DOI: 10.1016/j.fuel.2017.10.107

Hu B. y Zhai H. (2017). The cost of carbon capture and storage for coal-fired power plants in China. Int. J. Greenh. Gas. Con. 65 (1), 23-31.

DOI: 10.1016/j.ijggc.2017.08.009

IEAGHG (2014). $\mathrm{CO}_{2}$ capture at coal based power and hydrogen plants. Reporte. International Energy Agency Greenhouse Gas. Cheltenham, Reino Unido, 1028 pp.

IEA (2017). World energy outlook 2017. Reporte. International Energy Agency. París, Francia, 32 pp.

IPCC (2017). IPCC expert meeting on mitigation, sustainability and climate stabilization scenarios. Reporte. Intergovernmental Panel on Climate Change. Addis Ababa, Etiopía, 44 pp.

IPCC (2018). Summary for policymakers. En: Global warming of $1.5^{\circ} \mathrm{C}$ : An IPCC special report on the impacts of global warming of $1.5^{\circ} \mathrm{C}$ above pre-industrial levels and related global greenhouse gas emission pathways, in the context of strengthening the global response to the threat of climate change, sustainable development, and efforts to eradicate poverty (V. Masson-Delmotte, P. Zhai, H.O. Pörtner, D. Roberts, J. Skea, P.R. Shukla, A. Pirani, W. Moufouma-Okia, C. Péan, R. Pidcock, S. Connors, J.B.R. Matthews, Y. Chen, X. Zhou, M.I. Gomis, E. Lonnoy, T. Maycock, M. Tignor y T. Waterfield, Eds.). World Meteorological Organization, Génova, Suiza, 32 pp.

Le Quéré C., Andrew R.M., Friedlingstein P., Sitch S., Hauck J., Pongratz J., Pickers P.A., Korsbakken J. I., Peters G.P., Canadell J.G., Arneth A., Arora V.K., Barbero L., Bastos A., Bopp L., Chevallier F., Chini L.P., Ciais P., Doney S.C, Gkritzalis T., Goll D.S., Harris I., Haverd V., Hoffman F.M., Hoppema M., Houghton R.A., Hurtt G., Ilyina T., Jain A.K., Johannessen T., Jones C.D., Kato E., Keeling R.F., Goldewijk K.K.,
Landschützer P., Lefèvre N., Lienert S., Liu Z., Lombardozzi D., Metzl N., Munro D.R., Nabel J.E.M.S., Nakaoka S.-I., Neill C., Olsen A., Ono T., Patra P., Peregon A., Peters W., Peylin P., Pfeil B., Pierrot D., Poulter B., Rehder G., Resplandy L., Robertson E., Rocher M., Rödenbeck C., Schuster U., Schwinger J., Séférian R., Skjelvan I., Steinhoff T., Sutton A., Tans P.P., Tian H., Tilbrook B., Tubiello F.N., van der LaanLuijkx I.T., van der Werf G.R., Viovy N., Walker A.P., Wiltshire A.J., Wright R., Zaehle S. y Zheng B. (2018). Global carbon budget 2018, Earth Syst. Sci. Data, 10, 2141-2194. DOI: 10.5194/essd-10-2141-2018

López R., Menéndez M., Fernández C. y BernardoSánchez A. (2018). The effects of scale-up and coalbiomass blending on supercritical coal oxy-combustion power plants. Energy 148 (1), 571-584.

DOI: 10.1016/j.energy.2018.01.179

Manzolini G., Sanchez E., Rezvani S., Macchi E., Goetheer E.L.V. y Vlugt T.J.H. (2015). Economic assessment of novel amine based $\mathrm{CO}_{2}$ capture technologies integrated in power plants based on European Benchmarking Task Force methodology. Appl. Energy. 138 (1), 546-558. DOI: $10.1016 /$ j.apenergy.2014.04.066

Mardones C. y Muñoz T. (2017). Impuesto al $\mathrm{CO}_{2}$ en el sector eléctrico chileno: efectividad y efectos macroeconómicos. Rev. Econ. Chil. 20 (1), 4-25.

Mardones C. y Flores B. (2017). Evaluation of a $\mathrm{CO}_{2}$ tax in Chile: Emissions reduction or design problems? Lat. Am. Res. Rev. 52 (3), 334-343. DOI: 10.25222/larr.33

Mardones C. y Baeza N. (2018). Economic and environmental effects of a $\mathrm{CO}_{2}$ tax in Latin American countries. Energ. Policy. 114, 262-273.

DOI: $10.1016 / j$.enpol.2017.12.001

Mardones C. y Flores B. (2017). Effectiveness of a $\mathrm{CO}_{2}$ tax on industrial emissions. Energ. Econ. 71, 370-382. DOI: $10.1016 /$ j.eneco.2018.03.018

MMA (2017). Tercer reporte del estado del medio ambiente. Ministerio del Medio Ambiente. Reporte. Santiago, Chile, 198 pp.

MMA (2017). Plan de acción nacional de cambio climático 2017-2022. Ministerio del Medio Ambiente. Reporte. Santiago, Chile, 73-74 pp.

Osagie E., Biliyok C., Di Lorenzo G., Hanak D.P. y Manovic V. (2018). Techno-economic evaluation of the 2-amino-2-methyl-1-propanol (AMP) process for $\mathrm{CO}_{2}$ capture from natural gas combined cycle power plant. Int. J. Greenh. Gas. Con. 70 (1), 45-56.

DOI: 10.1016/j.ijggc.2018.01.010

Pettinau A., Ferrara F., Tola V. y Cau G. (2017). Technoeconomic comparison between different technologies for $\mathrm{CO}_{2}$-free power generation from coal. Appl. Energy. 193 (1), 426-439.

DOI: 10.1016/j.apenergy.2017.02.056 
Petrescu L., Bonalumi D., Valenti G., Cormos A.M. y Cormos C.C. (2017). Life cycle assessment for supercritical pulverized coal power plants with postcombustion carbon capture and storage. J. Clean. Prod. $157(1), 10-21$.

DOI: $10.1016 /$ j.jclepro.2017.03.225

Rochedo P.R.R., Costa I.V.L, Império M., Hoffmann B.S., Merschmann P.R., Oliveira C.C.N., Szklo A. y Schaeffer R. (2016). Carbon capture potential and costs in Brazil. J. Clean. Prod. 131 (1), 280-295.

DOI: 10.1016/j.jclepro.2016.05.033

Rubin E.S. y Zhai H. (2012). The cost of carbon capture and storage for natural gas combined cycle power plants. Environ. Sci. Technol. 46 (6), 3076-3084.

DOI: $10.1021 / \mathrm{es} 204514 \mathrm{f}$

SMA (2016). Instructivo para la cuantificación de las emisiones de fuentes fijas afectas al impuesto del artículo $8^{\circ}$ de la ley $n^{\circ} 20780$. Reporte. Superintendencia del Medio Ambiente. Santiago, Chile, 19-25 pp.

Singh U. y Rao A.B. (2016). Techno-economic assessment of carbon mitigation options for existing coal-fired power plants in India. Enrgy. Proced. 90 (1), 326-335. DOI: 10.1016/j.egypro.2016.11.200

USDOE (2013). Current and future technologies for natural gas combined cycle (NGCC) power plants. Reporte. United States Department of Energy. Pittsburg, EUA, 251 pp.
Valencia M.J. y Cardona C.A. (2013). Aproximación conceptual a la separación del dióxido de carbono en corrientes de combustión. Revista Facultad de Ingeniería 22 (34), 45-53. DOI: 10.19053/01211129.2217

Valiani S., Tahouni N. y Panjeshahi M.H. (2017). Optimization of pre-combustion capture for thermal power plants using Pinch Analysis. Energy 119 (1), 950-960. DOI: 10.1016/j.energy.2016.11.046

Van der Spek M., Sánchez E., Henrik N., Skagestad R., Ramirez A. y Faaij A. (2017). Unravelling uncertainty and variability in early stagetechno-economic assessments of carbon capture technologies. Int. J. Greenh. Gas. Con. 56 (1), 221-236.

DOI: 10.1016/j.ijggc.2016.11.021

Vera S. y Sauma E. (2015). Does a carbon tax make sense in countries with still a high potential for energy efficiency? Comparison between the reducing-emissions effects of carbon tax and energy efficiency measures in the Chilean case. Energy 88 (1), 478-488. DOI: $10.1016 /$ j.energy.2015.05.067

WMO (2018). The state of greenhouse gases in the atmosphere based on global observations through 2017. Reporte. World Meteorological Organization. Génova, Suiza, $1 \mathrm{pp}$.

ZEP (2011). The costs of $\mathrm{CO}_{2}$ capture, transport and storage. Reporte. Zero emissions platform. Bruselas, Bélgica, $50 \mathrm{pp}$. 


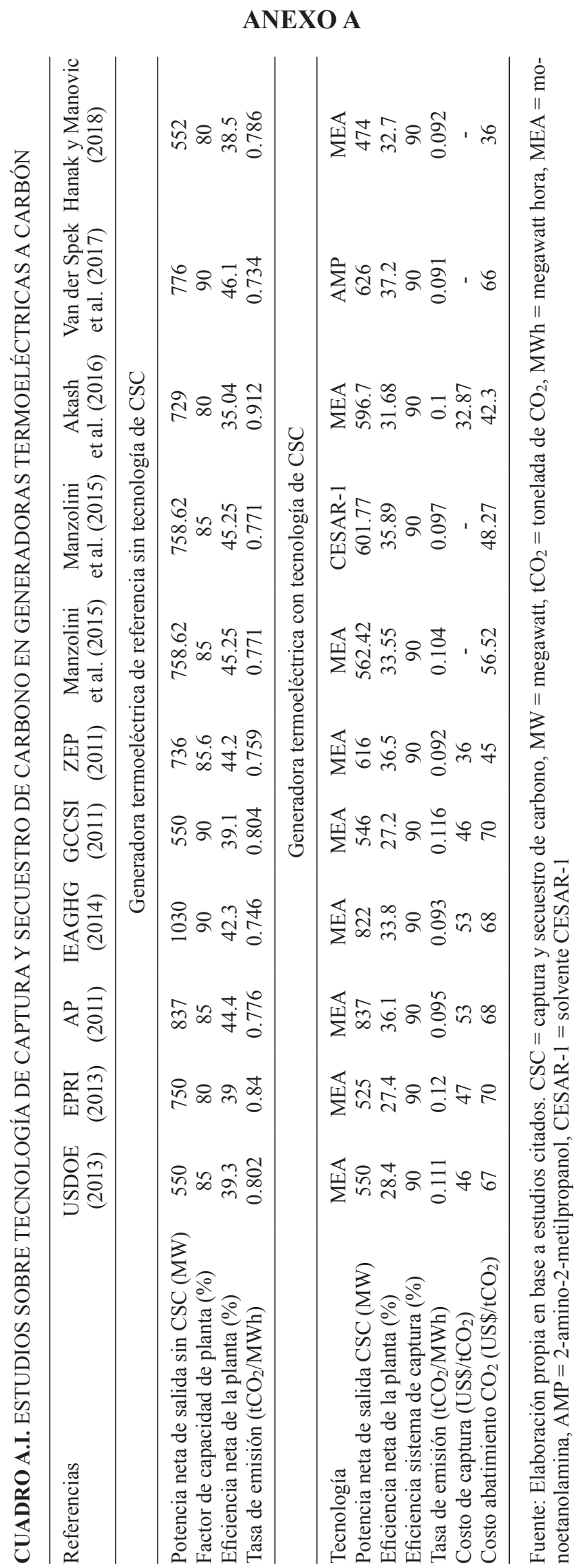




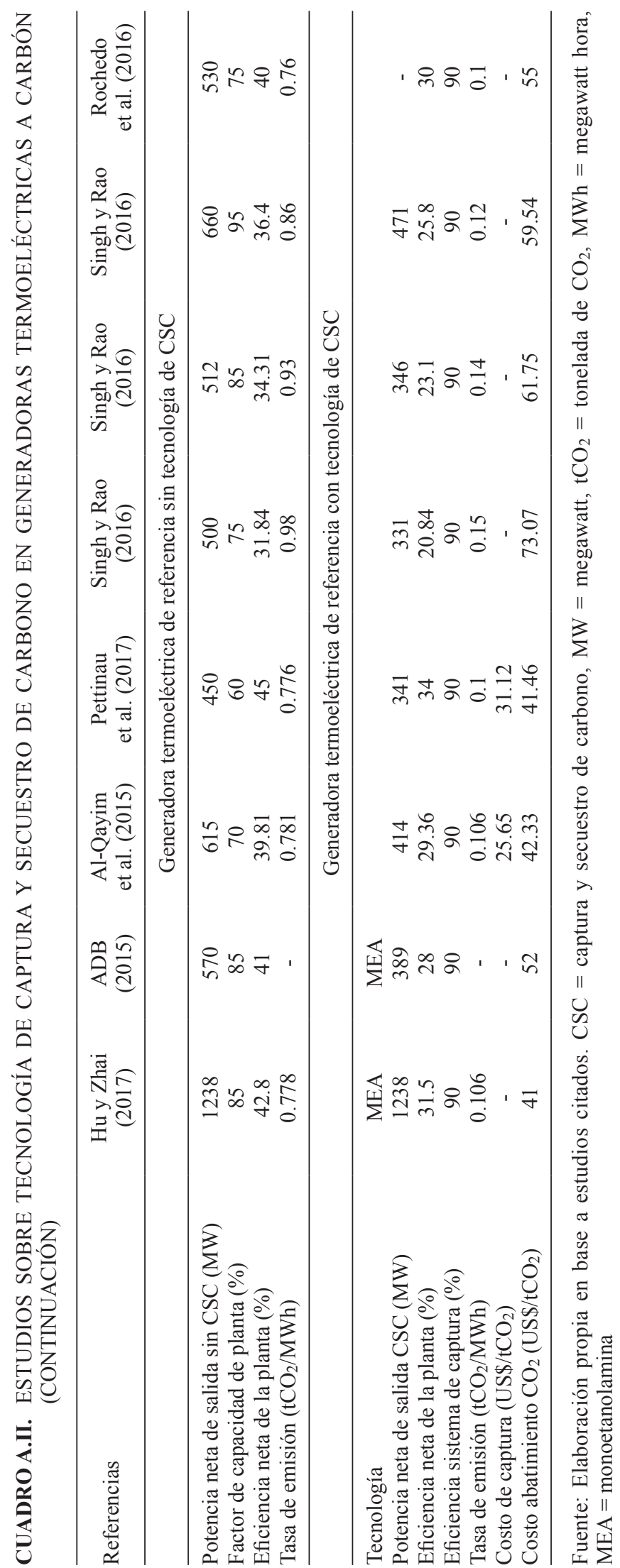




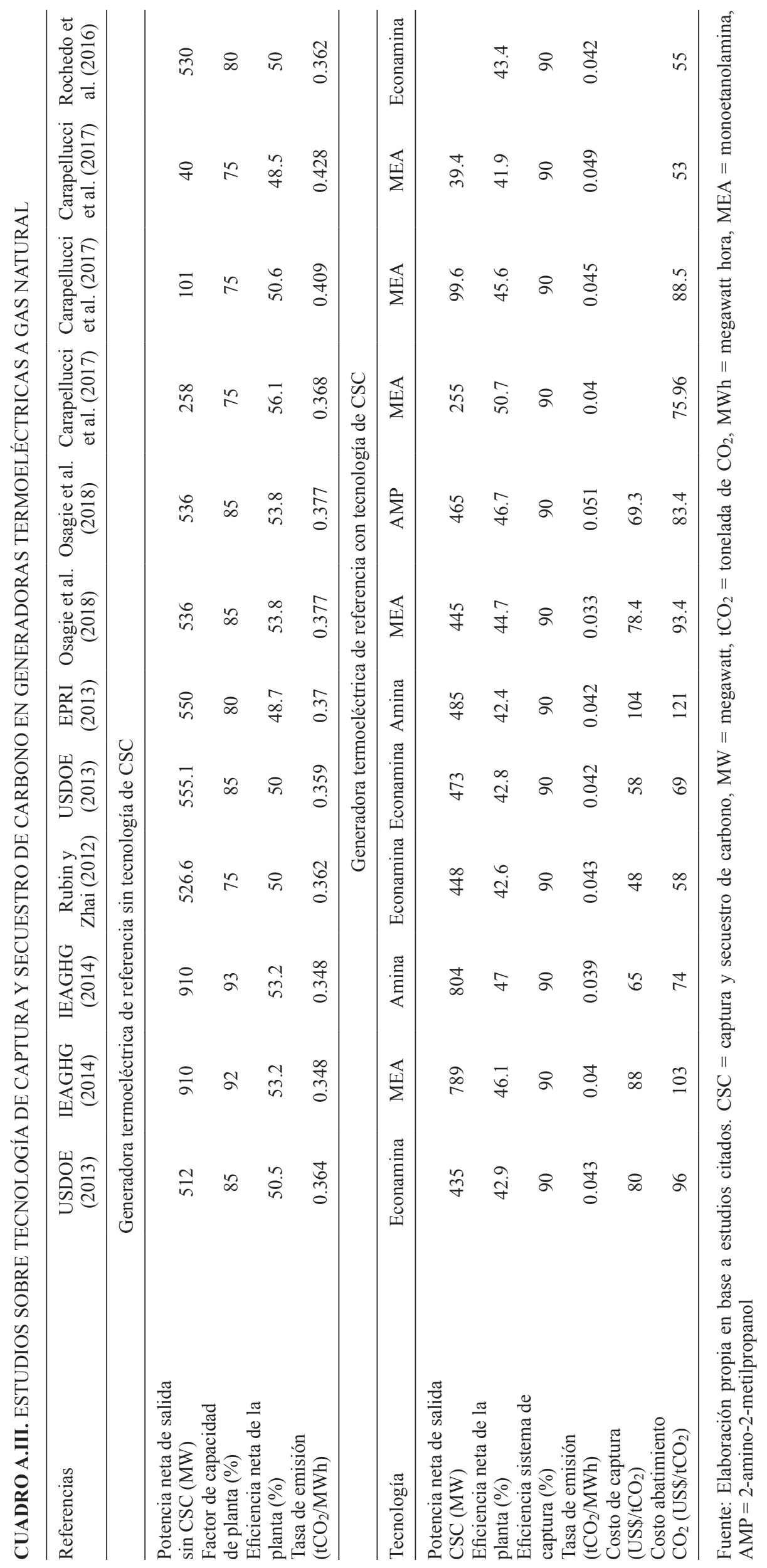




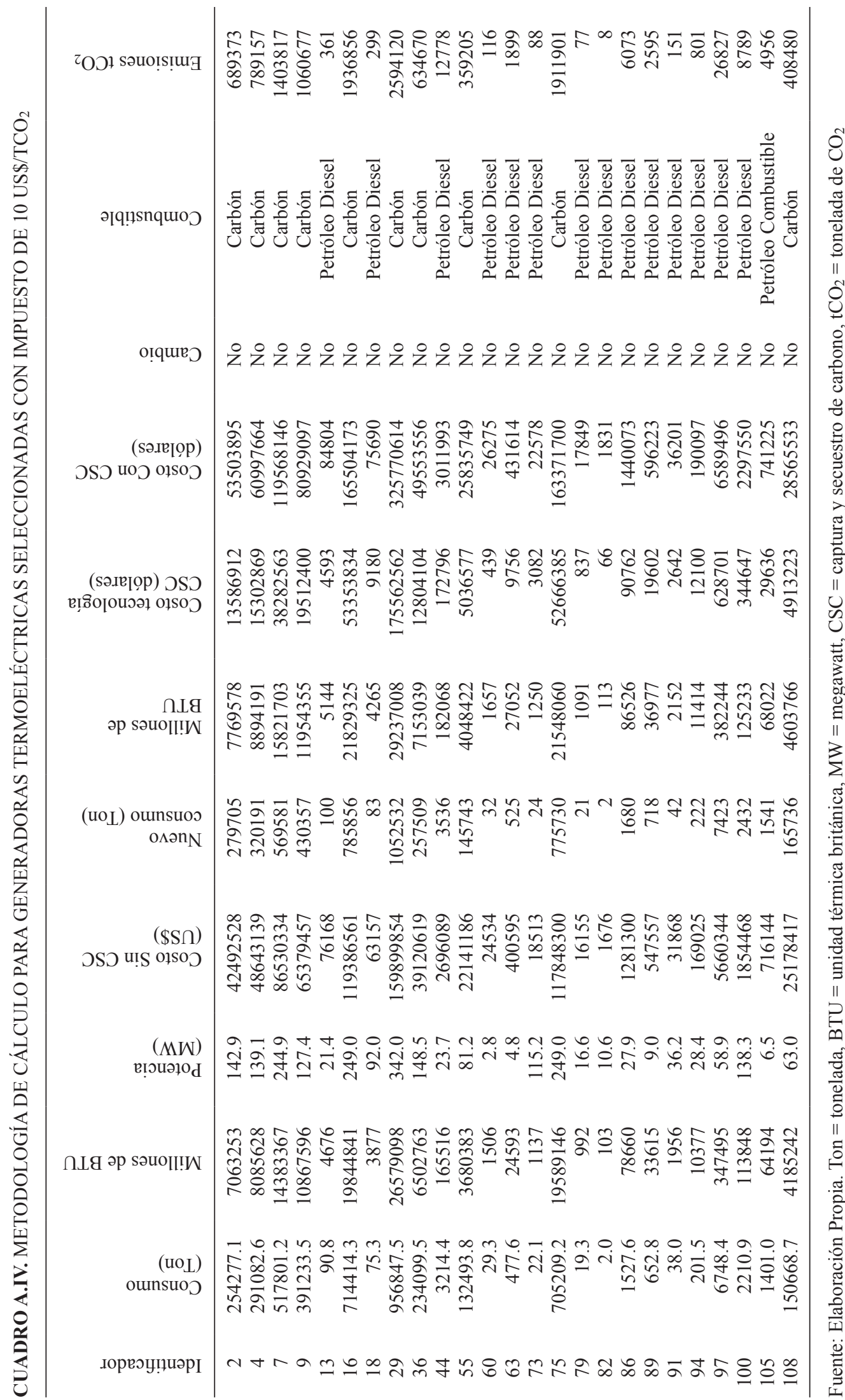




\section{ANEXO B}

CUADRO B.I. RESUMEN DE OBJETIVOS Y LÍNEAS DE ACCIÓN DEL PLAN DE ACCIÓN NACIONAL DE CAMBIO CLIMÁTICO (2017-2022)

\begin{tabular}{|c|c|c|}
\hline Eje temático & Objetivo específico & Líneas de acción (LA) \\
\hline \multirow{4}{*}{ Adaptación } & \multirow{2}{*}{$\begin{array}{l}\text { 3.1.1 Evaluar periódicamente la vulnerabilidad de siste- } \\
\text { mas humanos y naturales frente a los impactos del cambio } \\
\text { climático, estableciendo los riesgos y oportunidades que } \\
\text { presenta este fenómeno }\end{array}$} & $\begin{array}{l}\text { LA1: Generación, análisis y actualización de información } \\
\text { climática }\end{array}$ \\
\hline & & $\begin{array}{l}\text { LA2: Generación, análisis y actualización de información } \\
\text { sobre vulnerabilidad y riesgos frente cambio climático }\end{array}$ \\
\hline & $\begin{array}{l}\text { 3.1.2 Adaptarse al cambio climático, mediante la imple- } \\
\text { mentación de medidas dirigidas a reducir la vulnerabili- } \\
\text { dad y aumentar la capacidad adaptativa de los sistemas } \\
\text { humanos y naturales del país }\end{array}$ & $\begin{array}{l}\text { LA3: Elaboración, implementación y actualización de } \\
\text { planes sectoriales de adaptación }\end{array}$ \\
\hline & $\begin{array}{l}\text { 3.1.3 Monitorear y reportar periódicamente el avance de } \\
\text { la adaptación en el país, para establecer mejoras en la } \\
\text { planificación mediante políticas de adaptación }\end{array}$ & $\begin{array}{l}\text { LA4: Desarrollo de un sistema de monitoreo y reporte } \\
\text { para la adaptación en Chile }\end{array}$ \\
\hline \multirow{12}{*}{ Mitigación } & \multirow[t]{3}{*}{$\begin{array}{l}\text { 3.2.1 Mantener el Sistema Nacional de Inventarios de GEI } \\
\text { y actualizar el Inventario de Gases de Efecto Invernadero }\end{array}$} & $\begin{array}{l}\text { LA5: Actualización del Inventario de Gases de Efecto } \\
\text { Invernadero de Chile }\end{array}$ \\
\hline & & LA6: Sistema de garantía y control de calidad \\
\hline & & LA7: Archivo y difusión \\
\hline & \multirow{6}{*}{$\begin{array}{l}\text { 3.2.2. Desarrollar e implementar acciones y políticas de } \\
\text { mitigación }\end{array}$} & LA8: Acciones de mitigación del sector energía \\
\hline & & LA9: Acciones de mitigación del sector transporte \\
\hline & & $\begin{array}{l}\text { LA10: Acciones de mitigación del sector silvoagrope- } \\
\text { cuario }\end{array}$ \\
\hline & & $\begin{array}{l}\text { LA11: Acciones de mitigación para la edificación, ur- } \\
\text { banización e infraestructura pública bajas en carbono }\end{array}$ \\
\hline & & LA12: Acciones de mitigación del sector residuos \\
\hline & & $\begin{array}{l}\text { LA13: Acciones de mitigación transversales o multi- } \\
\text { sectoriales }\end{array}$ \\
\hline & $\begin{array}{l}\text { 3.2.3. Contabilizar y proponer medidas de mitigación de } \\
\text { contaminantes climáticos de vida corta }\end{array}$ & $\begin{array}{l}\text { LA14: Contabilidad y control de contaminantes climá- } \\
\text { ticos de vida corta }\end{array}$ \\
\hline & $\begin{array}{l}\text { 3.2.4 Implementar sistemas de contabilidad y monitoreo, } \\
\text { reporte y verificación }\end{array}$ & $\begin{array}{l}\text { LA15: Diseñar e implementar sistemas de monitoreo, } \\
\text { reporte y verificación para acciones de mitigación }\end{array}$ \\
\hline & $\begin{array}{l}\text { 3.2.5. Implementar acciones para cumplir con los com- } \\
\text { promisos internacionales de mitigación }\end{array}$ & $\begin{array}{l}\text { LA16: Desarrollar herramientas técnicas de análisis de } \\
\text { los compromisos de mitigación pre y pos- } 2020\end{array}$ \\
\hline
\end{tabular}

Fuente: MMA (2017) 
CUADRO B.I. RESUMEN DE OBJETIVOS Y LÍNEAS DE ACCIÓN DEL PLAN DE ACCIÓN NACIONAL DE CAMBIO CLIMÁTICO (2017-2022) (CONTINUACIÓN)

\begin{tabular}{|c|c|c|}
\hline Eje temático & Objetivo específico & Líneas de acción (LA) \\
\hline \multirow{8}{*}{$\begin{array}{l}\text { Medios de } \\
\text { implementación }\end{array}$} & $\begin{array}{l}\text { 3.3.1 Fortalecer la institucionalidad para abordar el cam- } \\
\text { bio climático a nivel nacional }\end{array}$ & LA17: Política y marco legal sobre cambio climático \\
\hline & \multirow{2}{*}{$\begin{array}{l}\text { 3.3.2 Apoyar la transferencia de tecnología para la im- } \\
\text { plementación de medidas de mitigación y adaptación al } \\
\text { cambio climático }\end{array}$} & $\begin{array}{l}\text { LA18: Estrategia para el desarrollo y la transferencia } \\
\text { tecnológica }\end{array}$ \\
\hline & & $\begin{array}{l}\text { LA19: Incorporación de nuevas tecnologías } \\
\text { LA24: Estrategia de educación }\end{array}$ \\
\hline & \multirow[t]{3}{*}{$\begin{array}{l}\text { 3.3.3 Crear las capacidades nacionales para la gestión del } \\
\text { cambio climático y entregar asistencia técnica }\end{array}$} & $\begin{array}{l}\text { LA20: Fomento de la investigación en temas de } \\
\text { cambio climático }\end{array}$ \\
\hline & & $\begin{array}{l}\text { LA21: Estrategia de educación y sensibilización para } \\
\text { abordar el cambio climático }\end{array}$ \\
\hline & & LA22: Cooperación Internacional \\
\hline & $\begin{array}{l}\text { 3.3.4 Generar una estrategia nacional financiera frente al } \\
\text { cambio climático }\end{array}$ & $\begin{array}{l}\text { LA23: Estrategia nacional financiera frente al cam- } \\
\text { bio climático }\end{array}$ \\
\hline & $\begin{array}{l}\text { 3.3.5 Asesorar la negociación internacional en temáticas } \\
\text { relacionadas al cambio climático }\end{array}$ & $\begin{array}{l}\text { LA24: Participación de Chile en la Convención } \\
\text { Marco de las Naciones Unidas sobre el Cambio } \\
\text { Climático, IPCC, Organización para la Cooperación } \\
\text { y el Desarrollo Económico y otros relacionados }\end{array}$ \\
\hline \multirow{6}{*}{$\begin{array}{l}\text { Gestión del cambio } \\
\text { climático a nivel } \\
\text { regional y comunal }\end{array}$} & \multirow{2}{*}{$\begin{array}{l}\text { 3.4.1 Fortalecer la institucionalidad de cambio climático } \\
\text { a nivel regional y comunal }\end{array}$} & LA25: Arreglos institucionales \\
\hline & & $\begin{array}{l}\text { LA26: Incorporación del cambio climático en los } \\
\text { instrumentos de planificación }\end{array}$ \\
\hline & \multirow[t]{3}{*}{$\begin{array}{l}\text { 3.4.2 Desarrollar las capacidades en los gobiernos regio- } \\
\text { nales y municipales }\end{array}$} & $\begin{array}{l}\text { LA27: Desarrollo de información a nivel regional y } \\
\text { comunal para apoyar la toma de decisiones }\end{array}$ \\
\hline & & $\begin{array}{l}\text { LA28: Desarrollo de programas de capacitación y } \\
\text { difusión }\end{array}$ \\
\hline & & $\begin{array}{l}\text { LA29: Fortalecer el sistema de certificación am- } \\
\text { biental municipal (SCAM) y el programa de barrios } \\
\text { sustentables }\end{array}$ \\
\hline & $\begin{array}{l}\text { 3.4.3 Implementar acciones y propiciar sinergias entre la } \\
\text { adaptación y la mitigación para un territorio específico }\end{array}$ & $\begin{array}{l}\text { LA30: Sinergias para la acción climática en un } \\
\text { territorio específico }\end{array}$ \\
\hline
\end{tabular}

